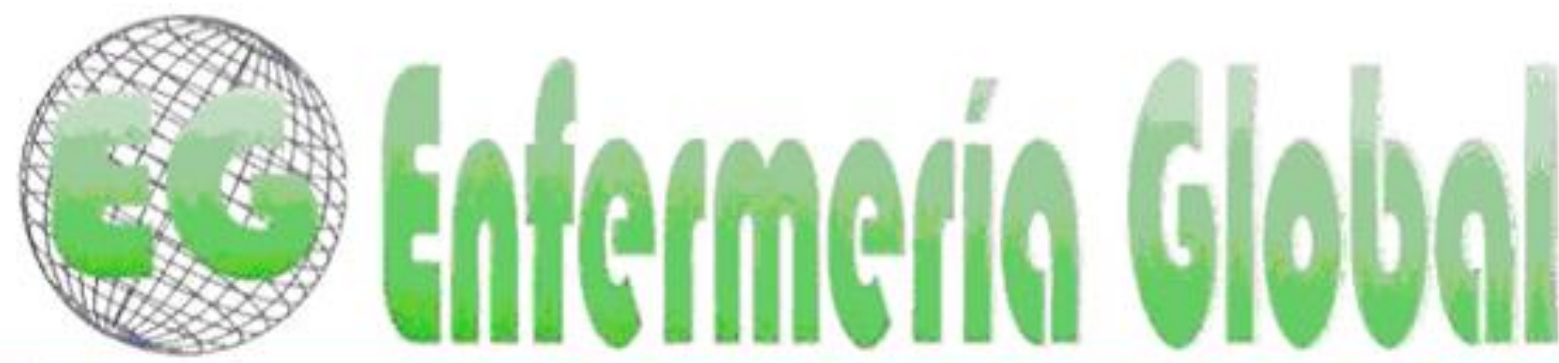

ISSN 1695-6141

Revista electrónica trimestral de Enfermeria

No34

Abril 2014

www.um.es/eglobal/

REVISIONES

\title{
Patologías de la comunicación. Proyecto docente para enfermería infantil. Dislalias
}

Communication pathology. Teaching project for child nursing. Dyslalias

\section{*Campos Palomo, Álvaro David **Campos Palomo, Laura Deseada}

\begin{abstract}
*Enfermero. Logopeda. Servicio de Urgencias. Hospital Universitario Virgen de la Victoria. Málaga. Email: marbillo86@hotmail.com **Enfermera. Servicio de Urgencias. Hospital Punta de Europa. Algeciras. Cádiz España.
\end{abstract}

Palabras clave: Comunicación; logopedia; dislalia; enfermería; patología articulatória Keywords: Articulation disorders; Communication;, dyslalia; nursing; speech therapy.

\section{RESUMEN}

La dislalia, mal conocida como trastorno del lenguaje, es sin duda una de las patologías de la comunicación, concretamente del habla, más común en la infancia. A pesar de que en los distintos servicios de salud en los que presta sus cuidados la profesión de enfermería se encuentran niños con estas dificultades, la formación profesional tanto pre como postgrado es muy deficiente en lo que a patología de la comunicación respecta.

Mediante una exhaustiva búsqueda bibliográfica actualizada en la Biblioteca Virtual del Sistema Público de Salud de Andalucía, se pretende dar a conocer los aspectos más relevantes de las dislalias con un objetivo púramente docente, lo que resultará en una mejora en los registros de enfermería, en el uso correcto de la terminología logopédica y en un avance en la coordinación interprofesional, consiguiendo con ello dar calidad e identidad a los cuidados de enfermería.

\section{ABSTRACT}

Dyslalia, bad known as a language disorder, is undoubtedly one of the most common childhood speech communication pathologies. Although nursing provide health care to children with these difficulties along different services, pregraduate and postgraduate training regarding communication pathology is very poor. Through an extensive updated searched literature in the Virtual Library System of Andalusia Public Health, is intended to present the most relevant aspects about dyslalia with pure teaching objective with the aim of improving nursing records, getting correct use in speech therapy terminology and achieving quality and identity in providing nursing care. 


\section{INTRODUCCIÓN}

La dislalia es la alteración en la pronunciación de uno o varios fonemas por alteración, sustitución u omisión de los mismos en personas que no poseen lesiones neurológicas centrales ni malformaciones en los órganos fonoarticulatorios. En estos casos, la estimulación temprana sería crucial para adquirir el contenido fonemático, lexicológico y sintáctico de la lengua ${ }^{(1)}$.

De los primeros trabajos que recupera la Biblioteca Virtual del Sistema Público de Andalucía sobre este tema, se puede citar el estudio realizado por Peacher WG y su equipo publicado tras finalizar la Segunda Guerra Mundial sobre los métodos de evaluación existentes en dislalias y disartrias. Desde entonces, y hasta hoy, el número de trabajos publicados en comparación a otras dificultades, como la afasia, es relativamente escaso, pero muestran un claro avance en la metodología de valoración e intervención en este trastorno articulatorio ${ }^{(2)}$.

En la mayoría de las ocasiones, estas investigaciones tanto de carácter primario como secundario, no son redactadas por profesionales enfermeros, sino por otro tipo de expertos que juegan un papel fundamental en el equipo multidisciplinar. Logopedas, pedagogos, psicólogos del lenguaje, neurólogos, maestros en audición y lenguaje, etc, son sólo algunas de las que se pueden mencionar. La literatura escrita por enfermería, aun tratando con pacientes que tienen dificultades articulatorias, es prácticamente nula.

La formación de enfermería debe ser continuada a lo largo de todo el ciclo profesional, y aprender de lenguaje, habla y comunicación en general, derivaría en calidad e identidad profesional en todos los cuidados que se presten.

Con el presente trabajo, no se pretende caer en el tópico del intrusismo. No es función de enfermería evaluar ni intervenir una dislalia, para eso existen otros profesionales formados específicamente en trastornos del lenguaje, el habla, la voz, la lectoescritura y/o la sordera, como por ejemplo, la figura del logopeda, tan útil como imprescindible en todo tipo de servicios sanitarios o de educación donde haya niños con cualquier trastorno de la comunicación.

El objetivo principal de este estudio descriptivo, consiste en dar a conocer a aquellas enfermeras que trabajan en el área de infantil, así como a los estudiantes de la disciplina, un conocimiento básico sobre la definición y clasificación de la dislalia, consiguiendo así optimizar el uso de la terminología logopédica en los registros profesionales, mejorar la coordinación interprofesional en el abordaje multidisciplinar y holístico del niño y derivando todo ello en la mejora de la calidad de vida de los pacientes.

\section{MATERIAL Y MÉTODOS}

El presente trabajo descriptivo se ha realizado mediante una exhaustiva y actualizada búsqueda bibliográfica que se detalla a continuación.

- Estrategia de búsqueda

- Metabuscador utilizado: 
.GERIÓN (Biblioteca Virtual del Sistema Sanitario Público de Andalucía). Utilizando la búsqueda simple y avanzada en bases de datos de enfermería.

Bases de datos consultadas a través del metabuscador:

Agencia española del medicamento y productos sanitarios

ISOC - Humanidades y Ciencias Sociales (CSIC)

. Cuidatge. Referències bibliogràfiques en Infermeria

. Practica de Cuidados de salud. Joanna Briggs

. New England Journal of Medicine (NEJM)

. ScienceDirect - Journals- V.4 (Elsevier)

. ICYT - Ciencia y Tecnología (CSIC)

. CUIDEN. Base de Datos de Enfermería

. Lippincott'S Nursing Procedures

. CINHAL

. Elsevier.es (antiguo Doyma)

. Enfispo

. HighWire Press

. IBECS

. IME- Biomedicina

. Instituto Joanna Briggs

. Nursing@Ovid

OvidMD

. OvidSP

. PEDro

Pubmed

. SciELO España

. Wiley-Blackwell - Journals

- Ecuación de búsqueda. Primera criba.

- Todos los términos utilizados tanto en la primera como en la segunda criba, están incluidos en los Medical Subject Headings (términos MeSH).

- Para esta primera criba se utilizó la búsqueda simple de GERIÓN en bases de datos de enfermería.

- Términos MeSH utilizados por separado:

1. "speech disorder"

2. "articulation disorder"

- En suma, GERIÓN recuperó 274 artículos de un total de 160.069 encontrados.

- Ecuación de búsqueda. Segunda criba.

- Para esta segunda criba se utilizó la búsqueda avanzada de GERIÓN, en bases de datos de enfermería y utilizando "todos los campos" y "título" en el motor de búsqueda. 
. Términos MeSH utilizados:

1. "speech perception"

2. "speech acoustics"

3. "phonetics"

4. "speech disorder"

5. "articulation disorder"

Desarrollo de las ecuaciones

1. "speech disorder" AND "speech perception"

2. "articulaton disorder" AND "speech acoustics"

3. "articulation disorder" AND "phonetics"

. En suma, GERIÓN recuperó 180 trabajos de un total de 13.917 encontrados que se sometieron a los siguientes criterios de inclusión/exclusión de artículos.

- Criterios de inclusión de artículos

- Artículos publicados en el periodo de tiempo 2008 - 2012

- Artículos con resúmenes completos en castellano y/o inglés con definiciones claras y conclusiones específicas

- Implicación de la profesión enfermera en alguna vertiente. Clínica, docente, investigadora o gestora.

- Estudios centrados en dislalia

- Criterios excepcionales de inclusión de artículos

- Aquellos artículos/manuales que sin cumplir los requisitos de inclusión, se han considerado relevantes por los autores en materia de definición, marco teórico o histórico del presente trabajo.

- Criterios de exclusión de artículos

- Trabajos con escasa o nula evidencia científica.

- Abordaje puramente logopédico o pedagógico.

- Artículos de opinión.

- Cualquier estudio que no cumpla los requisitos primarios o excepcionales de inclusión.

- Maniobra de extracción de datos

- Se seleccionaron 26 artículos/manuales que cumplían los objetivos del estudio y se consideraron relevantes en la búsqueda bibliográfica, así como en la comprensión de la dislalia infantil en enfermería.

- Para la extracción de datos, se utilizaron las siguientes variables secundarias en inglés (términos "no MeSH").

. "Dislalia" 
. "phoneme"

. Y los nombres propios de las alteraciones dislálicas, como por ejemplo "rotacism", "sigmatism", etc

- Maniobra de referencia de datos a lo largo del trabajo.

- Se utilizaron las directrices de la revista Enfermería Global y las normas de Vancouver (última revisión en abril 2010).

\section{RESULTADOS}

- Clasificación integradora de la dislalia (Adaptación de Gallego) ${ }^{(3)}$

- Dislalia evolutiva: Propia de la inmadurez neuronal y considerada normal hasta los seis años, periodo en el que la prevalencia de dificultades articulatorias es más común en niños (16,7\%) que en niñas (12,7\%), siendo la educación paterna, número de hermanos o género, los factores más concluyentes en la dinámica articulatoria y sus patologías. Además cabe destacar que los niños con trastorno fonológico poseen una tasa de velocidad de habla normalmente reducida, hecho no significativo en las consultas de niño sano que atienden los profesionales de enfermería a diario en este rango de edad ${ }^{(4,5)}$.

- Dislalia audiógena: En este caso, el niño presenta dificultad en la discriminación auditiva básica. Enfermería debe conocer que la memoria, la fonología y la inteligibilidad del habla es igual en los niños con trastorno específico del lenguaje y trastorno del procesamiento auditivo y que el rol del logopeda en el diagnóstico y tratamiento de este último continúa bajo discusión, difiriendo mucho de los estudios individuales y la práctica clínica, recomendándose continuar la investigación para el desarrollo de una evaluación correcta así como derivaciones e intervenciones de calidad. Se puede resaltar además la importancia de la enfermería escolar y el equipo multidisciplinar en la detección precoz de trastornos auditivos ${ }^{(6-10)}$.

- Dislalia orgánica: Se produce cuando la lesión se encuentra producida por una alteración de los órganos periféricos del habla. Es la comúnmente llamada por otros autores, disglosia. A la consulta de niño sano, normalmente llegan tras la intervención quirúrgica que se realiza en los primeros meses de vida y la enfermera se encuentra en un punto ideal para derivar al paciente al logopeda para su posterior evaluación y/o intervención en caso necesario, ya que, el defecto en la articulación que produce una disglosia, puede interferir en el proceso de aprendizaje posterior ${ }^{(11)}$.

- Dislalia funcional: Producida por el uso inapropiado de los órganos fonoarticulatorios. Consiste en la dificultad que posee el niño para encontrar el punto de articulación correcto para producir el fonema deseado, pudiendo incluso producir, en casos severos, alteraciones en la atención e hiperactividad, como demostró Lewis BA en un estudio sobre esta temática. Los endofenotivos y factores genéticos, suponen otra línea de investigación importante al respecto. Influyen notablemente en la producción sonora en los primeros años de infancia, y en la edad escolar, a nivel de lectura, ortografía, 
lenguaje hablado y expresiones escritas. Todos estos trabajos realizados, poco a poco se van teniendo en cuenta en la práctica clínica profesional, y el logopeda, al igual que la enfermera, necesitan de su experiencia para incluir dichos hallazgos en su bagaje de conocimientos, teniendo además en cuenta factores como las preferencias o valores del paciente ${ }^{(12-14)}$.

- Denominación de la dislalia

- Como especifica Pascual García en su amplísima y dilatada experiencia con la dislalia, denominaremos al trastorno según el fonema afectado, usando una terminología griega terminada en "cismo" o "tismo". Así hablaremos de: (15)

. Rotacismo: Dificultad en la pronunciación de /r/

. Ceceo: Pronunciación de /s/por /z/

. Jotacismo: Dificultad en la pronunciación del fonema / $\mathrm{x} /$

. Ficismo: La no articulación del fonema /f/

. Seseo: Pronunciación de /z/ por/s/

. Numación: La no articulación del fonema/n/

. Kappacismo: Dificultad en la pronunciación del fonema /k/

. Sigmatismo: Dificultad en la pronunciación del fonema /s/

- Lambdacismo: Dificultad en la articulación del fonema ///

. Mitacismo: La no articulación del fonema $/ \mathrm{m} /$

. Nunación: la no articulación del fonema /ñ/

. Piscismo: Dificultad en la articulación del fonema /p/

- Gammacismo: Dificultad en la pronunciación del fonema /g/

. Chuitismo: la no articulación del fonema /ch/

- Tetacismo: La no articulación del fonema /t/

. Yeismo: Dificultad en la articulación del fonema /II/

- Cuando un fonema es sustituido por otro, se antepone "para" en la denominación de la dislalia. Por ejemplo, un niño que pronuncia "dosa" en vez de "rosa", estaría ante un pararrotacismo ${ }^{(15)}$.

- Sintomatología de la dislalia

Se dan aquí una información muy escueta a modo resumen para que la enfermera tenga un mínimo conocimiento y sepa utilizar la terminología correctamente a la hora de derivar a un niño al logopeda para su posterior evaluación e intervención.

- Distorsión: Producción de sonidos de forma incorrecta o deformada y es mucho más frecuentes en los casos de baja conciencia fonológica ${ }^{(16)}$.

- Sustitución: Error de articulación en el que un sonido es correctamente pronunciado dentro de una palabra, pero no es el adecuado, hecho que se explica por la facilidad de emisión y fluidez que proporciona dicho fonema. Como ejemplo se puede poner la pronunciación de "cada" por "cara" ${ }^{(17) .}$

- Omisión: Eliminación del fonema que produce dificultad articulatoria, como por ejemplo pronunciar "andía" por "sandía" (18). 
- Inserción o adición: Introducción de un nuevo fonema para agilizar la pronunciación del fonema que presenta dificultad. Como ejemplo se podría poner la pronunciación de "daragón" por "dragón" ${ }^{(19)}$.

- Evaluación e intervención terapéutica de la dislalia

Se presenta aquí, a modo de conocimiento muy general para las enfermeras, cómo será el abordaje logopédico a niños dislálicos.

- Evaluación de la dislalia:

- Mediante pruebas de lenguaje estandarizadas y baremadas como el Registro Fonológico Inducido (RFI) de Monfort o PAF de Vallés ${ }^{(20,21)}$

Mediante protocolos estandarizados de evaluación de movimientos de los órganos bucofonatorios

. Mediante pruebas de discriminación de sonidos ${ }^{(22)}$

Algunos logopedas incluyen pruebas generales de fonología y praxias orofaciales así como de discriminación auditiva ${ }^{(22)}$

- La conducta, la motivación y la atención son otros aspectos a tener en cuenta y que posiblemente requieran intervención de otros profesionales ${ }^{(23)}$

Intervención terapéutica en dislalia: El esquema a seguir quedaría de la siguiente manera ${ }^{(24,25)}$ :

- Discriminación auditiva de los sonidos de la vida real y discriminación fonética de los sonidos del lenguaje con el objetivo de introducir en el desarrollo fonético/fonológico el sonido ausente.

- Ejercicios de los órganos bucofonatorios para aumentar la flexibilidad de los mismos.

- Tratamiento del fonema afectado empezando por un tratamiento aislado del mismo y evolucionando a la generalización del mismo.

. Corrección de movimientos linguales incorrectos.

- Generalización con el objetivo de integrar los nuevos sonidos correctos y adquiridos en la vida cotidiana.

Adecuación al caso concreto, teniendo en cuenta factores de desarrollo emocional, habilidades sociales, edad, entorno, etc.

\section{DISCUSIÓN}

La dislalia es una patología del habla, que no del lenguaje, muy común en las consultas de niño sano que atienden las enfermeras a diario en los distintos centros de salud de la amplia red sanitaria española. Con el presente trabajo, se han incorporado unos conocimientos básicos de la dislalia a lo que viene siendo el quehacer diario de los profesionales en los citados servicios, y, como se viene argumentando a lo largo de todo el proyecto, aunque la responsabilidad de evaluar e intervenir estos niños es del logopeda, no está de más adquirir conocimientos al respecto, ya que, tanto la formación pregrado como la postgrado, es muy deficiente en lo que a patologías del lenguaje, habla, voz, lectoescritura y comunicación en general respecta. 


\section{CONCLUSIONES}

Los autores consideramos que la información arrojada puede ser de extrema utilidad si enfermería introduce en sus planes de cuidados la Educación para la Salud (EpS) a padres de niños dislálicos, aportando esta publicación una vía de información a los mismos que garantizaría una calidad e identidad profesional en los cuidados de enfermería, ya que, como se ha venido reseñando, las alteraciones del habla podrían interferir en los trastornos de hiperactividad y atención ${ }^{(26)}$.

Sobre estas líneas, abordando la utilidad de este análisis descriptivo de la dislalia, y dejando a un lado la EpS, el aumento de vocabulario adquirido mejoraría las relaciones interprofesionales entre enfermería y logopedia, al usar una terminología muy desconocida por el gremio de expertos cuidadores, de una manera correcta.

\section{REFERENCIAS BIBLIOGRÁFICAS}

1. Labrada Sánchez I. Actividades para la preparación a la familia en el desarrollo de la pronunciación de los sonidos del lenguaje en un niño de cuatro a cinco años atendido por el programa educa a tu hijo. Cuadernos de Educación y Desarrollo 2011; 3(23)

2. Peacher WG., Peacher GM. Speech disorders in World War II; dysarthria and dyslalia; methods of examination. J Nerv Ment Dis 1946; 103: 484-93

3. Gallego OJ. Calidad en la intervención logopédica. Estudio de Casos. Málaga. Aljibe. 1999

4. Karbasi SA., Fallah R., Golestam M. The prevalence of speech disorder in primary school students in Yazd-Iran. Acta Médica Iránica 2011; 49(1): 33-7.

5. Wertzner HF., Silva LM. Speech rate in children with and without phonological disorder. Profono 2009; 21(1): 19-24

6. Ferguson MA., Hall RL., Riley A., Moore DR. Communication, listening, cognitive and speech perception skills in children with auditory processing disorder (apd) or specific language impairment (sli). Journal of Speech, Language \& Hearing Research 2011; 54(1): 211-27

7. Richard GJ. The Role of the Speech-Language Pathologist in identifying and Treating Children With Auditory Processing Disorder. Language, Speech \& Hearing Services in Schools 2011; 42(3): 241-5

8. Debonis DA., Moncrieff D. Auditory Processing Disorders: An Update for Speech-Language Pathologists. Am J Speech Lang Pathol 2008; 17(1): 4-18

9. Neville K., Foley M., Gertner A. Understanding and identifying the child at risk for auditory processing disorders: a case method approach in examining the interdisciplinary role of the school nurse. Journal of School Nursing (Sage Publications Inc.) 2011; 27(1): 22-23

10. Campbell N. Supporting children with auditory processing disorder. British Journal of School Nursing 2011; 6(6): 273-7

11. Khamis-Dakwar R., Froud K., Gordon P. Acquiring diglossia: mutual influences of formal and colloquial Arabic on children's grammaticality judments. Journal of Child Language 2012; 39(1): 61-89

12. Lewis BA., Short EJ., lyengar SK., Taylor HG., Freebairn L., Tag J., Avrich AA., Stein CM. Speech-Sound Disorders and Attention-Deficit/Hiperactivity Disorder Symptoms. Topics in Languaje Disorders 2012; 32(3): 247-63. 
13. Baker E., Sharynne M. Evidence- Based Practice for Children With Speech Sound Disorders: Part 2 Application to Clinical Practice. Lang Speech Hear Serv Sch 2011; 42(2): 140-151

14. Barbara AL., Allison AV., Lisa AF., Amy JH., Lara ES., Iris K., Taylor HG., Suda KL., Catherine MS. Literacy Outcomes of Children With Early Childhood Speech Sound Disorders: Impact of Endophenotypes. J Speech Lang hear Res 2011; 54(6): 1628-1643

15. Pascual García P. La dislalia. Madrid. CEPE. 2012

16. Preston J., Edwards ML. Phonological Awareness and Types of Sound Errors in Preschoolers With Speech Sound Disorders. J Speech Lang Hear res 2010; 53(1): 44-60.

17. Kirk C. Substitution Errors in the Production of Word-Initial and Word-Final Consonant Clusters. J Speech Lang Hear Res 2008; 51: 35-48.

18. Cohen-Mimran R. The Contribution of Language Skills to Reading Fluency: A Comparison of two Orthographies for Hebrew. Journal of child Languaje 2009; 36(3): 657-672

19. Hogan TP. A Short Report: Word-Level Phonological and lexical Characteristics Interact to Influence Phoneme Awareness. Journal of Learning Disabilities 2010; 43(4): 346-356

20. Vallés Arándiga A. PAF: Evaluación de la dislalia 3a Ed. Madrid CEPE. 2010

21. Monfort M. Juárez A. Registro Fonológico Inducido. Madrid. CEPE. 2006

22. Bustos I. Discriminación fonética y auditiva. Madrid. CEPE. 1990

23. Hurtado Gómez MJ. Trastornos del lenguaje. Revista Digital Innovación y Experiencias Educativas 2009; 16

24. Obregón Sanz S. E.AR Enseñanza de la articulación 2ª Ed. Madrid. CEPE. 2006

25. Pascual García P. La dislalia. Naturaleza, diagnóstico y rehabilitación. $3^{\text {a }}$ Ed. Madrid. CEPE. 2008

26. Tomblin JB., Mueller K. Foreword: Exploring the Comorbidity of AttentionDeficit/Hyperactivity Disorder and Languaje, Speech, and Reading Disorders. Topics in Languaje Disorders 2012; 32(3): 195-97. 